\title{
A Study on Multipeutics
}

\section{Jens Christian Larsen}

Vanløse Alle 50 2. mf. tv, 2720 Vanløse, Copenhagen, Denmark

Email: jlarsen.math@hotmail.com

How to cite this paper: Larsen, J.C. (2017) A Study on Multipeutics. Applied Mathematics, 8, 746-773.

https://doi.org/10.4236/am.2017.85059

Received: April 3, 2017

Accepted: May 24, 2017

Published: May 27, 2017

Copyright $\odot 2017$ by author and Scientific Research Publishing Inc. This work is licensed under the Creative Commons Attribution International License (CC BY 4.0).

http://creativecommons.org/licenses/by/4.0/

\begin{abstract}
Multipeutics is the simultaneous application of $m \geq 4$ cancer treatments. $m=$ 4 is quadrapeutics, which was invented by researchers at Rice University, Northeastern University, MD Anderson Cancer Centre and China Medical University, see [1]. Multipeutics is our idea. From section 6 Summary, it follows that multipeutics can be more potent than quadrapeutics by comparing these two mathematical models. The first two treatments in quadrapeutics are systemically administered nano gold particles $G$ and lysosomal chemo therapeutic drug D. They form mixed clusters $M$ primarily in cancer cells and can be excited by a laser pulse, the third treatment, to form plasmonic nanobubbles $\mathrm{N}$. These nanobubbles can kill the cancer cells by mechanical impact. If they do not the chemo therapeutic drug can be released into the cytoplasm, which might be lethal to the cancer cell. The fourth treatment is $\mathrm{x}$ rays $\mathrm{X}$ and the cancer cells have been sensitized to $\mathrm{x}$ rays by the treatment. We present an ODE (ordinary differential equations) model of quadrapeutics and of multipeutics, which is quadrapeutics and $n \geq 1$ immune or chemo therapies. In the present paper we have found a polynomial $p$ of degree at most $2(n+3)$, such that a singular point $\left(C, D, G, M, N, I_{1}, \cdots, I_{n}\right)$ will have $p(M)=0$ Here $I_{1}, \cdots, I_{n}$ are immune or chemo therapies. So this gives us candidates for singular points. Quadrapeutics is treated extensively. We find in theorem 3 a polynomium $s$ of degree at most six in $M$ such that a positive singular point $(C, D, G$, $M, N)$ of the quadrapeutics system will have $s(M)=0$. The main theorem of the present paper is the multipeutics theorem, saying that the more treatments we apply the lower the cancer burden, even if we take the doses of each treatment smaller. From the proof of this theorem, we can say, that quadrapeutics can outperform chemo radiation if the nanobubble kill rate $k_{21}$ is sufficiently big. See also Figure 1 and Figure 2 and the text explaining them.
\end{abstract}

\section{Keywords}

Cancer, Mass Action Kinetic System, Quadrapeutics, Immune Therapy 


\section{Introduction}

In [2] we introduced a discrete three dimensional model of cancer growth and a three dimensional ODE model of cancer growth. The variables are $C$ cancer cells, $G F$ growth factors (positive control) and growth inhibitors $G I$ (negative control) in both models. In [3] we showed that there are cancer ODE models with oscillatory behaviour and in [4] we found a discrete mathematical model of $C, G F, G I$ with a nontrivial attractor. In the same paper, we introduced mathematical models of the four phases of adoptive $\mathrm{T}$ cell therapy. The first phase is the resection of the tumor and lymphodepletion with a chemo therapeutic drug. The second phase is the pre REP phase where the TILs (tumor infiltrating lymphocytes) from the patients' tumor are expanded with IL-2 treatment. The third phase is the REP phase of expanding and activating the TILs with IL-2 and anti CD3. The fourth phase is the injection of the expanded and activated TIL s back into the patient. We showed that this last phase of the treatment is bistable in the following sense. There is a positive sink and there exist initial conditions such that the cancer burden goes to infinity as $t \rightarrow+t^{+}$, where $] t^{-}, t^{+}\left[t^{-}<0, t^{+}>0\right.$ is the domain of definition of the maximal integral curve $c(t)$ of the corresponding vector field.

In the present paper, we propose to combine quadrapeutics and immune therapy.

We have written a paper [5] on fundamental concepts in dynamics intended for researchers with a background in medicine.

There are several important monographs related to the present paper, see [6]-[11]. [12] is a mathematical model of miRNAs. [13] [14] [15] are papers on quadrapeutics. [16] [17] are two papers of mine on cancer and mathematics.

\section{The Multipeutics System}

Consider the mass action kinetic system

$$
\begin{aligned}
& C+N \rightarrow 0 \\
& C+D \rightarrow 0 \\
& D+G \rightarrow M \\
& M \rightarrow N \\
& N \rightarrow 0 \\
& M \rightarrow 0 \\
& C+X \rightarrow 0 \\
& C \rightarrow 2 C \\
& C \leftrightarrows 0 \\
& D \leftrightarrows 0 \\
& G \leftrightarrows 0 \\
& C+I_{i} \rightarrow 0 \quad i=1, \cdots, n \\
& I_{i} \leftrightarrows 0
\end{aligned}
$$


$n \in \mathbb{N}_{0} \quad(n=0$ means that the reactions (12) and (13) are omitted). The complexes are $C(1)=C+N, C(2)=0, \quad C(3)=C+D, C(4)=D+G, \quad C(5)=M$, $C(6)=N, \quad C(7)=C+X, \quad C(8)=C, \quad C(9)=2 C, \quad C(10)=D, \quad C(11)=G$, $C(10+2 i)=C+I_{i}, \quad C(11+2 i)=I_{i}, i=1, \cdots, n$ The variables here are $C$ cancer cells, $D$ lysosomal chemo therapeutic drug, $G$ nanogold particles conjugated to an antibody panitumumab against the epidermal growth factor receptor $E G F R$, $M$ mixed clusters in cancer cells, $N$ nanobubbles and finally $I_{i}$ immune or chemo therapies. $X$, the $\mathrm{x}$ rays, is not a variable but a parameter, a positive real number. (1) says that nanobubbles kill cancer cells and (2) that the chemo therapeutic drug kills cancer cells. (3) means that drug and nano gold particles form mixed clusters in cancer cells and normal cells and the cluster size is the largest in cancer cells. The mixed clusters generate nano bubbles (4), when excited with a laser pulse. The laser threshold pulse decreases with cluster size and since the cluster size is the largest in cancer cells it is mainly in cancer cells that plasmonic nanobubbles kill the host cell. (7) is the killing of cancer cells by $\mathrm{x}$ rays. (5) and (6) are decay rates for N and M. (8) says that cancer cells proliferate rapidly. (9), (10) and (11) give birth and decay rates for cancer cells, chemotherapeutic drug and nano gold particles, respectively. (12) and (13) are immune or chemo therapies. The rate constant in (7) is $\tilde{k}_{27}=k_{27} X$. The ODEs are, see [18]

$$
\begin{aligned}
& C^{\prime}=-k_{21} C \cdot N-k_{23} C \cdot D+\left(k_{98}-k_{28}-k_{27} X\right) C+k_{82}-\sum_{i=1}^{n} k_{2,10+2 i} C \cdot I_{i} \\
& D^{\prime}=-k_{23} C \cdot D-k_{54} D \cdot G+k_{10,2}-k_{2,10} D \\
& G^{\prime}=-k_{54} D \cdot G+k_{11,2}-k_{2,11} G \\
& M^{\prime}=k_{54} D \cdot G-\left(k_{65}+k_{25}\right) M \\
& N^{\prime}=-k_{21} C \cdot N+k_{65} M-k_{26} N \\
& I_{i}^{\prime}=-k_{2,10+2 i} C \cdot I_{i}+k_{11+2 i, 2}-k_{2,11+2 i} I_{i} \quad i=1, \cdots, n
\end{aligned}
$$

The corresponding vector field is denoted $f$. We are going to consider subsystems of (14) to (19). First of all the chemo radiation system, the vector field in (14) and (15) with the rate constants in (16) to (19) equal to zero and the rest positive. This vector field is denoted

$$
f_{c r}: \mathbb{R}^{2} \rightarrow \mathbb{R}^{2}
$$

The quadrapeutics system (14) to (18) with the rate constants in (19) equal to zero and the rest positive. This vector field is denoted

$$
f_{q}: \mathbb{R}^{5} \rightarrow \mathbb{R}^{5}
$$

Finally the multipeutics systems: the system (14) to (19) with the rate constants in (19) equal to zero, when $i=n-j+1, \cdots, n$ and the rest positive. This vector field is denoted

$$
f_{n-j}: \mathbb{R}^{n-j+5} \rightarrow \mathbb{R}^{n-j+5}
$$

$j \in\{0, \cdots, n\}$. Now introduce some important notation. A singular point of $f_{c r}$ is denoted $c_{c r}=\left(C_{*}^{c r}, D_{*}^{c r}\right)$. A singular point of $f_{q}$ is denoted $c_{q}=$ 
$\left(C_{*}^{q}, D_{*}^{q}, G_{*}^{q}, M_{*}^{q}, N_{*}^{q}\right)$. A singular point of $f_{n-j}$ is denoted

$$
c_{n-j}=\left(C_{*}^{n-j}, D_{*}^{n-j}, G_{*}^{n-j}, M_{*}^{n-j}, N_{*}^{n-j}, I_{*}^{1, n-j}, \cdots, I_{*}^{n-j, n-j}\right)
$$

We are going to compare the cancer burden for the different systems. In fact we shall show that there are stable equilibria of the different systems such that

$$
C_{*}^{n}<C_{*}^{n-1}<\cdots<C_{*}^{1}<C_{*}^{q}<C_{*}^{c r}
$$

Looking from right to left, this says that the more treatments we apply the lower the cancer burden.

In Figure 1 we have plotted $C(t)$ versus $t$ for the chemo radiation model and $C(t)$ versus $t$ for the quadrapeutics model. All rate constants equal to 1 , except $k_{21}=10, k_{65}=10, k_{11,2}=20$. Also $X=1$. This supports the proof of Theorem 1 that if you make the nanobubble kill rate $k_{21}$ sufficiently big, then quadrapeutics outperforms chemo radiation. I have iterated the Euler maps

$$
\begin{aligned}
& H_{c r}(C, D)=(C, D)+h f_{c r}(C, D) \\
& H_{q}(C, D, G, M, N)=(C, D, G, M, N)+h f_{q}(C, D, G, M, N)
\end{aligned}
$$

where $h$ is the step size and equal to 0.02 . There are 1000 iterations. The numerical analysis indicated, that $C(t), D(t), G(t), M(t), N(t)$ for the quadrapeutics system converged, when $t$ goes to infinity. And similarly for $C(t), D(t)$ of the chemo radiation model, see Figure 1 and Figure 2.

Theorem 1. Assume, that $\hat{a} \triangleq k_{98}-k_{28}-k_{27} X<0$. There exist positive values of the rate constants $k_{i j}$, such that (24) holds and the singular points are all positive and stable.

Proof. Let $K$ be defined by

$$
\begin{aligned}
& k_{1}=\left(k_{82}, k_{10,2}, k_{11,2},\left\{k_{11+2 i, 2}\right\}_{i=1, \cdots, n}\right) \\
& k_{2}=\left(k_{21}, k_{23}, k_{98}, k_{28}, \tilde{k}_{27}, k_{54}, k_{2,10}, k_{2,11}, k_{65}, k_{25}, k_{26},\left\{k_{2,10+2 i}, k_{2,11+2 i}\right\}_{i=1, \cdots, n}\right) \\
& K=\left(k_{1}, k_{2}\right)
\end{aligned}
$$

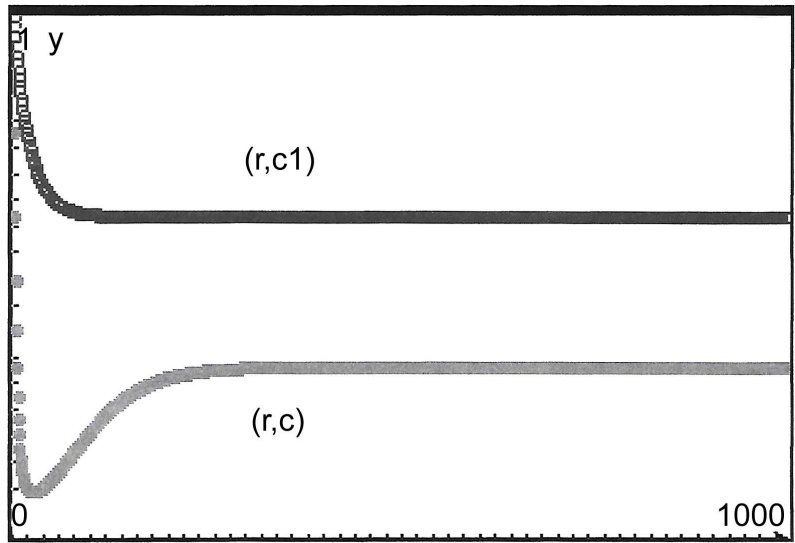

Figure 1. $(\mathrm{r}, \mathrm{cl})$ is $C(t)$ versus $t$ for the chemo radiation model and $(\mathrm{r}, \mathrm{c})$ is $C(t)$ versus $t$ for the quadrapeutics model. 


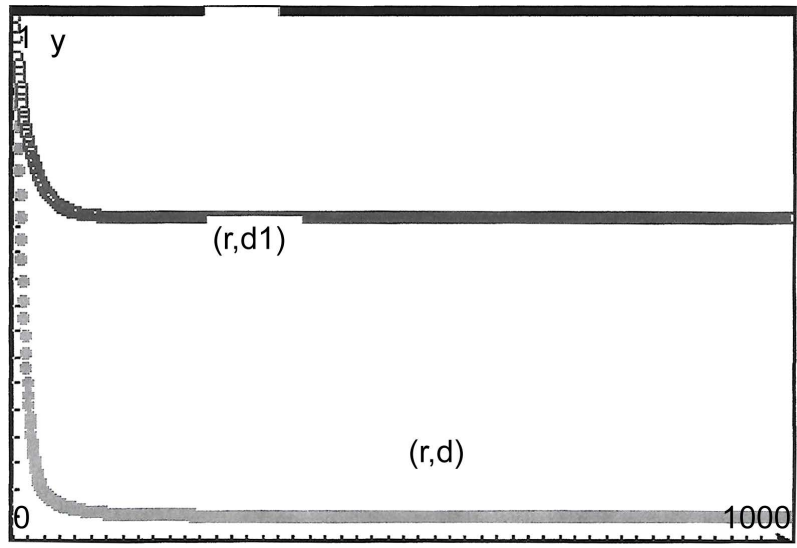

Figure 2. (r, d1) is $D(t)$ versus $t$ for the chemo radiation system and (r, d) is $D(t)$ versus $t$ for the quadrapeutics system.

Define

$$
\begin{aligned}
& k_{82}=t k_{82}^{0} \\
& k_{10,2}=t k_{10,2}^{0} \\
& k_{11,2}=t k_{11,2}^{0} \\
& k_{11+2 i, 2}=t k_{11+2 i, 2}^{0} \quad i=1, \cdots, n \quad t \in \mathbb{R}
\end{aligned}
$$

when $*=n$. Here

$$
k_{82}^{0}, k_{10,2}^{0}, k_{11,2}^{0}, k_{11+2 i, 2}^{0} \in \mathbb{R}_{+}
$$

$i=1, \cdots, n$. Notice that $(0, \cdots, 0)$ is a singular point of $f$, when $t=0$. The rate constants $k_{i j}$ in $k_{2}$ are positive real numbers. Also note that $D_{1} f_{*_{0}}$ is an isomorphism.

Here

$$
*=\left\{\begin{array}{c}
c r \\
q \\
1 \\
\vdots \\
n
\end{array}\right.
$$

For instance in $f$

$$
D_{1} f_{0}=\left\{b_{i j}\right\}_{i, j=1, \cdots, n}
$$

is an isomorphism, where $b_{11}=\hat{a}, b_{2,2}=-k_{2,10}, b_{3,3}=-k_{2,11}, b_{4,4}=-\left(k_{65}+k_{25}\right)$, $b_{5,5}=-k_{26}, \quad b_{5,4}=k_{65}, \quad b_{66}=-k_{2,13}, \cdots, b_{n+5, n+5}=-k_{2,11+2 n}$, all other $b_{i j}=0$.

So there exist $C^{k}$ maps, $k \geq 5$,

$$
C_{*}: V_{*} \subset \mathbb{R}^{p_{*}} \rightarrow \mathbb{R}^{s_{*}}
$$

such that

$$
f_{*}\left(c_{*}\left(K_{*}\right), K_{*}\right)=0 \quad c_{*}\left(\hat{K}_{*}\right)=(0, \cdots, 0)
$$

by the implicit function theorem. $V_{*}, K_{*}, \hat{K}_{*}$ are defined below. 
Also

$$
\begin{aligned}
& p_{c r}=7 \quad s_{c r}=2 \\
& p_{q}=14 \quad s_{q}=5 \\
& p_{n-j}=14+3(n-j) \quad s_{n-j}=5+n-j
\end{aligned}
$$

For $*=c r$ define

$$
k_{1}^{c r}=\left(k_{82}, k_{10,2}\right)
$$

and

$$
k_{2}^{c r}=\left(k_{23}, k_{98}, k_{28}, \tilde{k}_{27}, k_{2,10}\right)
$$

For $*=q$ we define

$$
k_{1}^{q}=\left(k_{82}, k_{10,2}, k_{11,2}\right)
$$

and

$$
k_{2}^{q}=\left(k_{2}^{c r}, k_{21}, k_{2,11}, k_{54}, k_{65}, k_{25}, k_{26}\right)
$$

Finally for the multipeutics system

$$
k_{1}^{n-j}=\left(k_{82}, k_{10,2}, k_{11,2}, k_{11+2 i, 2 i=1, \cdots, n-j}\right)
$$

and

$$
k_{2}^{n-j}=\left(k_{2}^{q},\left(k_{2,10+2 i}, k_{2,11+2 i}\right)_{i=1, \cdots, n-j}\right)
$$

Now let $\hat{K}_{c r}=\left(k_{1}^{c r}, k_{2}^{c r}\right), k_{1}^{c r}=0$ and $\hat{K}_{q}=\left(k_{1}^{q}, k_{2}^{q}\right), k_{1}^{q}=0$, and finally $\hat{K}_{n-j}=\left(k_{1}^{n-j}, k_{2}^{n-j}\right), k_{1}^{n-j}=0 . \quad V_{*}$ above is an open subset containing $\hat{K}_{*}$ in $\mathbb{R}^{p^{*}}$. Define $K_{*}=\left(k_{1}^{*}, k_{2}^{*}\right)$. Then

$$
f_{*}\left(c_{*}\left(t k_{1}^{*, 0}, k_{2}^{*}\right), t k_{1}^{*, 0}, k_{2}^{*}\right)=0
$$

Define

$$
d_{*}(t)=c_{*}\left(t k_{1}^{*, 0}, k_{2}^{*}\right)
$$

For the chemo radiation model we let

$$
d_{*}^{G}=0 \quad d_{*}^{M}=0 \quad d_{*}^{N}=0 \quad d_{*}^{I_{i}}=0
$$

$i=1, \cdots, n$. For the quadrapeutics model we let $d_{*}^{I_{i}}=0, i=1, \cdots, n$. And for the multipeutics model we let

$$
d_{*}^{I_{i}}=0 \quad i=n-j+1, \cdots, n \quad j \in\{0, \cdots, n\}
$$

Differentiate (48) with respect to $t$ to get

$$
D_{1} f\left(\frac{\partial d_{*}}{\partial t}\right)+\frac{\partial f}{\partial t}=0
$$

Thus

$$
\frac{\partial d_{*}^{C}}{\partial t}(0)=-\frac{1}{\hat{a}} k_{82}^{0}
$$

regardless of the system. We have 


$$
-k_{21} d_{*}^{C} d_{*}^{N}-k_{23} d_{*}^{C} d_{*}^{D}+\left(k_{98}-k_{28}-k_{27} X\right) d_{*}^{C}+k_{82}-\sum_{i=1}^{n} k_{2,10+2 i} d_{*}^{C} d_{*}^{I_{i}}=0
$$

Differentiate this with respect to $t$ to get

$$
\begin{aligned}
& -k_{21}\left(\frac{\partial d_{*}^{C}}{\partial t} d_{*}^{N}+d_{*}^{C} \frac{\partial d_{*}^{N}}{\partial t}\right)-k_{23}\left(\frac{\partial d_{*}^{C}}{\partial t} d_{*}^{D}+d_{*}^{C} \frac{\partial d_{*}^{D}}{\partial t}\right) \\
& +\hat{a} \frac{\partial d_{*}^{C}}{\partial t}+k_{82}^{0}-\sum_{i=1}^{n} k_{2,10+2 i}\left(\frac{\partial d_{*}^{C}}{\partial t} d_{*}^{I_{i}}+d_{*}^{C} \frac{\partial d_{*}^{I_{i}}}{\partial t}\right)=0
\end{aligned}
$$

Also differentiate this equation with respect to $t$ to find

$$
\begin{aligned}
& -k_{21}\left(\frac{\partial^{2} d_{*}^{C}}{\partial t^{2}} d_{*}^{N}+2 \frac{\partial d_{*}^{C}}{\partial t} \frac{\partial d_{*}^{N}}{\partial t}+d_{*}^{C} \frac{\partial^{2} d_{*}^{N}}{\partial t^{2}}\right) \\
& -k_{23}\left(\frac{\partial^{2} d_{*}^{C}}{\partial t^{2}} d_{*}^{D}+2 \frac{\partial d_{*}^{C}}{\partial t} \frac{\partial d_{*}^{D}}{\partial t}+d_{*}^{C} \frac{\partial^{2} d_{*}^{D}}{\partial t^{2}}\right) \\
& -\sum_{i=1}^{n} k_{2,10+2 i}\left(\frac{\partial^{2} d_{*}^{C}}{\partial t^{2}} d_{*}^{I_{i}}+2 \frac{\partial d_{*}^{C}}{\partial t} \frac{\partial d_{*}^{I_{i}}}{\partial t}+d_{*}^{C} \frac{\partial^{2} d_{*}^{I_{i}}}{\partial t^{2}}\right) \\
& +\hat{a} \frac{\partial^{2} d_{*}^{C}}{\partial t^{2}}=0
\end{aligned}
$$

From this equation it follows that

$$
\frac{\partial^{2} d_{c r}^{C}}{\partial t^{2}}(0)=\frac{\partial^{2} d_{q}^{C}}{\partial t^{2}}(0)>\frac{\partial^{2} d_{1}^{C}}{\partial t^{2}}(0)>\cdots>\frac{\partial^{2} d_{n}^{C}}{\partial t^{2}}(0)
$$

anticipating that

$$
d_{*}^{N}(0)=0 \quad \frac{\partial d_{*}^{N}}{\partial t}(0)=0
$$

Observe that

$$
\begin{gathered}
\frac{\partial d_{*}^{C}}{\partial t}(0)=-\frac{k_{82}^{0}}{\hat{a}}>0 \quad \frac{\partial d_{*}^{D}}{\partial t}(0)=\frac{k_{10,2}^{0}}{k_{2,10}}>0 \\
\frac{\partial d_{*}^{G}}{\partial t}(0)=\frac{k_{11,2}^{0}}{k_{2,11}}>0 \quad \frac{\partial d_{*}^{I_{i}}}{\partial t}(0)=\frac{k_{11+2 i, 2}^{0}}{k_{2,11+2 i}}>0
\end{gathered}
$$

$i=1, \cdots, n$. The third equality holds for $*=q, 1, \cdots, n$. The fourth equality holds for the multipeutics system $f_{n-j}, i=1, \cdots, n-j, j \in\{0,1, \cdots, n\}$.

By the fundamental theorem of calculus applied twice

$$
d_{*}^{C}(t)=\int_{0}^{t}\left(\int_{0}^{s} \frac{\partial^{2} d_{*}^{C}}{\partial t^{2}}(v) \mathrm{d} v+\frac{\partial d_{*}^{C}}{\partial t}(0)\right) \mathrm{d} s
$$

So

$$
d_{q}^{C}(t)>d_{1}^{C}(t)>\cdots>d_{n}^{C}(t)
$$

To see the first inequality, observe that there exists a positive constant $c$, such that 


$$
d_{q}^{C}(t)-d_{1}^{C}(t)=\int_{0}^{t} \int_{0}^{s}\left(\frac{\partial^{2} d_{q}^{C}}{\partial t^{2}}(v)-\frac{\partial^{2} d_{1}^{C}}{\partial t^{2}}(v)\right) \mathrm{d} v \mathrm{~d} s \geq \frac{1}{2} c t^{2}>0
$$

because a positive function on a compact interval $[0, \delta]$ assumes a minimum $c>0$. The other inequalities follow in the same way. And this proves all the inequalities in (24) except $C_{*}^{q}<C_{*}^{c r}$. Henceforth assume

$$
k_{2,10+2 i}=k_{11+2 i, 2}=k_{2,11+2 i}=0
$$

$i=1, \cdots, n$. Finally differentiate (57) to (60) with respect to $t$ to get

$$
\begin{aligned}
& -3 k_{21}\left(\frac{\partial^{2} d_{*}^{C}}{\partial t^{2}} \frac{\partial d_{*}^{N}}{\partial t}+\frac{\partial d_{*}^{C}}{\partial t} \frac{\partial^{2} d_{*}^{N}}{\partial t^{2}}\right) \\
& -3 k_{23}\left(\frac{\partial^{2} d_{*}^{C}}{\partial t^{2}} \frac{\partial d_{*}^{D}}{\partial t}+\frac{\partial d_{*}^{C}}{\partial t} \frac{\partial^{2} d_{*}^{D}}{\partial t^{2}}\right) \\
& +\hat{a} \frac{\partial^{3} d_{*}^{C}}{\partial t^{3}}=0
\end{aligned}
$$

when $t=0$. But

$$
\frac{\partial d_{*}^{M}}{\partial t}(0)=\frac{\partial d_{*}^{N}}{\partial t}(0)=0
$$

However when $*=q$

$$
\frac{\partial^{2} d_{*}^{N}}{\partial t^{2}}(0)>0
$$

To see this differentiate (17) twice with respect to $t$ to find

$$
\left(k_{65}+k_{25}\right) \frac{\partial^{2} d_{*}^{M}}{\partial t^{2}}(0)=2 k_{54} \frac{\partial d_{*}^{D}}{\partial t}(0) \frac{\partial d_{*}^{G}}{\partial t}(0)>0
$$

If we now differentiate (18) twice with respect to $t$ we find

$$
k_{26} \frac{\partial^{2} d_{*}^{N}}{\partial t^{2}}(0)=k_{65} \frac{\partial^{2} d_{*}^{M}}{\partial t^{2}}(0)>0
$$

which is what we sought to show.

Furthermore

$$
\left(k_{65}+k_{25}\right) d_{*}^{M}(t)=k_{54} d_{*}^{D} \cdot d_{*}^{G}(t)>0
$$

and

$$
d_{*}^{N}(t)=\frac{k_{65} d_{*}^{M}(t)}{k_{21} d_{*}^{C}(t)+k_{26}}>0
$$

when $t>0$ and small. Differentiate (15) twice with respect to $t$ when $*=c r, q$ to get

$$
-k_{2,10} \frac{\partial^{2} d_{*}^{D}}{\partial t^{2}}=2 k_{23} \frac{\partial d_{*}^{C}}{\partial t} \frac{\partial d_{*}^{D}}{\partial t}+2 k_{54} \frac{\partial d_{*}^{D}}{\partial t} \frac{\partial d_{*}^{G}}{\partial t}
$$

Now

$$
\hat{a} \frac{\partial^{2} d_{*}^{C}}{\partial t^{2}}=2 k_{23} \frac{\partial d_{*}^{C}}{\partial t} \frac{\partial d_{*}^{D}}{\partial t}
$$


Also

$$
\begin{aligned}
\hat{a} \frac{\partial^{3} d_{*}^{C}}{\partial t^{3}}(0)= & 3 k_{21}\left(\frac{\partial d_{*}^{C}}{\partial t} \frac{k_{65} k_{54} 2}{k_{26}\left(k_{65}+k_{25}\right)} \frac{\partial d_{*}^{D}}{\partial t} \frac{\partial d_{*}^{G}}{\partial t}\right) \\
& +3 k_{23} \frac{1}{\hat{a}}\left(2 k_{23} \frac{\partial d_{*}^{C}}{\partial t} \frac{\partial d_{*}^{D}}{\partial t}\right) \frac{\partial d_{*}^{D}}{\partial t} \\
& -3 k_{23} \frac{1}{k_{2,10}} \frac{\partial d_{*}^{C}}{\partial t}\left(2 k_{23} \frac{\partial d_{*}^{C}}{\partial t} \frac{\partial d_{*}^{D}}{\partial t}+2 k_{54} \frac{\partial d_{*}^{D}}{\partial t} \frac{\partial d_{*}^{G}}{\partial t}\right)
\end{aligned}
$$

where we have evaluated in $t=0$ on the right hand side. So if

$$
3 \frac{k_{21} k_{65} 2}{k_{26}\left(k_{65}+k_{25}\right)}-\frac{3 k_{32} 2}{k_{2,10}}>0
$$

we have

$$
\frac{\partial^{3} d_{c r}^{C}}{\partial t^{3}}(t)>\frac{\partial^{3} d_{q}^{C}}{\partial t^{3}}(t)
$$

when $t=0$ and hence also for small $t>0$. The fundamental theorem of calculus applied three times gives

$$
d_{*}^{C}(t)=\int_{0}^{t}\left(\int_{0}^{s}\left(\int_{0}^{v} \frac{\partial^{3} d_{*}^{C}}{\partial t^{3}}(u) \mathrm{d} u+\frac{\partial^{2} d_{*}^{C}}{\partial t^{2}}(0)\right) \mathrm{d} v+\frac{\partial d_{*}^{C}}{\partial t}(0)\right) \mathrm{d} s
$$

So

$$
d_{c r}^{C}(t)>d_{q}^{C}(t)
$$

for small $t>0$ and the theorem follows in the same way as (67). Define the matrices

$$
B=D f_{*_{*}}-\lambda \text { id } \quad A=D f_{*_{*}}
$$

and then write the definition of the determinant

$$
\operatorname{det} B=\sum_{\sigma \in S^{p}}(-1)^{I(\sigma)} B_{1, \sigma(1)} \cdots B_{p, \sigma(p)}
$$

where $S^{p}$ is the set of permutations of $\{1, \cdots, p\}$ and $I(\sigma)=0$ if $\sigma$ is even and $I(\sigma)=1$, when $\sigma$ is odd. This formula shows immediately that

$$
\operatorname{det} B=(-1)^{p} \lambda^{p}+\sum_{i=0}^{p-1} a_{i}\left(A_{11}, \cdots, A_{p p}\right) \lambda^{i}
$$

where $a_{i}$ is a polynomial in $A_{11}, \cdots, A_{p p}$, and hence is smooth in $A_{11}, \cdots, A_{p p}$. You can prove this by induction. Now apply the continuous dependence of roots of a polynomial on its coefficients to show that $d_{*}(t)$ is stable for $f_{*}$, when $t$ is small, see [19].

Remark. If the quadrapeutics chemo rate is

$$
\left.k_{10,2}^{q}=k_{10,2}^{c r} \tilde{\alpha} \quad \tilde{\alpha} \in\right] 0,1[
$$

and the $\mathrm{x}$ ray rate is

$$
\left.k_{27}^{q} X=k_{27}^{c r} X \tilde{\beta} \quad \tilde{\beta} \in\right] 0,1[
$$


where

$$
k_{10,2}^{q}=t k_{10,2}^{q, 0} \quad k_{10,2}^{c r}=t k_{10,2}^{c r, 0}
$$

while $\hat{a}=k_{98}-k_{28}-k_{27} X<0$. Here

$$
k_{10,2}^{q, 0}, k_{10,2}^{c r, 0} \in \mathbb{R}_{+}
$$

Then by taking $k_{21}$ big we find

$$
\frac{\partial^{3} d_{q}^{C}}{\partial t^{3}}(0)<\frac{\partial^{3} d_{c r}^{C}}{\partial t^{3}}(0)
$$

since

$$
\hat{a} \frac{\partial^{3} d_{q}^{C}}{\partial t^{3}}(0) \rightarrow+\infty
$$

as $k_{21} \rightarrow+\infty$. Hence

$$
d_{q}^{C}(t)<d_{c r}^{C}(t)
$$

for small $t>0$, when we impose (90) and (91), arguing as in the proof of theorem 1 .

Experimentally this is what you see, that quadrapeutics can outperform chemo radiation even if we take the chemo radiation doses smaller, see [1] [13] [14] [15].

Consider also the system (1) to (13) with

$$
2 C+X \rightarrow 0
$$

replacing (7). The ODE s are the same (15) to (19) except (14), which is

$$
C^{\prime}=-k_{21} C \cdot N-k_{23} C \cdot D+\left(k_{98}-k_{28}\right) C+k_{82}-2 k_{27} X \cdot C^{2}-\sum_{i=1}^{n} k_{2,10+2 i} C \cdot I_{i}
$$

We are now going to find candidates of the positive singular points of the different systems. We start with $f$ and assume that all $k_{i j}$ are positive. (19) gives

$$
I_{i}=\frac{k_{11+2 i, 2}}{k_{2,10+2 i} C+k_{2,11+2 i}}
$$

when $C>0$ and (18) gives

$$
N=\frac{k_{65} M}{k_{21} C+k_{26}}
$$

(16) and (17) give

$$
k_{11,2}-k_{2,11} G-\left(k_{65}+k_{25}\right) M=0
$$

or

$$
G=\frac{1}{k_{2,11}}\left(k_{11,2}-\left(k_{65}+k_{25}\right) M\right)=\alpha M+\beta
$$

Also

$$
D=\frac{k_{10,2}}{k_{23} C+k_{54} G+k_{2,10}}
$$


Insert these formulas in (14)

$$
\begin{aligned}
& -k_{21} C \frac{k_{65} M}{k_{21} C+k_{26}}-k_{23} C \frac{k_{10,2}}{k_{23} C+k_{54} G+k_{2,10}} \\
& +\left(k_{98}-k_{28}-k_{27} X(1+\delta(2 C-1))\right) C \\
& -\sum_{i=1}^{n} k_{2,10+2 i} C \frac{k_{11+2 i, 2}}{k_{2,10+2 i} C+k_{2,11+2 i}}+k_{82}=0
\end{aligned}
$$

$\delta=0,1$. Insert the formula for $D$ in $M^{\prime}=0$ to get

$$
k_{54} G \frac{k_{10,2}}{k_{23} C+k_{54} G+k_{2,10}}-\left(k_{65}+k_{26}\right) M=0
$$

We can isolate $C$ in this equation to find

$$
C=\frac{a M^{2}+b M+c}{M}
$$

where

$$
a=\left(k_{65}+k_{25}\right) \frac{k_{54}}{k_{2,11} k_{23}}
$$

and

$$
b=-\frac{k_{54} k_{10,2}}{k_{23} k_{2,11}}-\left(\frac{k_{54}}{k_{2,11} k_{23}} k_{11,2}+\frac{k_{2,10}}{k_{23}}\right)
$$

Finally

$$
c=\frac{k_{54} k_{10,2} k_{11,2}}{k_{23} k_{2,11}\left(k_{65}+k_{25}\right)}
$$

We now get by multiplying with the product of the denominators in (104), (105) and (106)

$$
\begin{aligned}
& -k_{21} C k_{65} M\left(k_{23} C+k_{54} G+k_{2,10}\right) \prod_{i=1}^{n}\left(k_{2,10+2 i} C+k_{2,11+2 i}\right) \\
& -k_{23} C k_{10,2}\left(k_{21} C+k_{26}\right) \prod_{i=1}^{n}\left(k_{2,10+2 i} C+k_{2,11+2 i}\right) \\
& +\left(k_{82}+\left(k_{98}-k_{28}-k_{27} X(1+\delta(2 C-1))\right) C\right)\left(k_{21} C+k_{26}\right) \\
& \cdot\left(k_{23} C+k_{54} G+k_{2,10}\right) \prod_{i=1}^{n}\left(k_{2,10+2 i} C+k_{2,11+2 i}\right) \\
& -\sum_{i=1}^{n} k_{2,10+2 i} C k_{11+2 i, 2}\left(\prod_{j=1, j \neq i}^{n}\left(k_{2,10+2 j} C+k_{2,11+2 j}\right)\right) \\
& \cdot\left(k_{23} C+k_{54} G+k_{2,10}\right)\left(k_{21} C+k_{26}\right)=0
\end{aligned}
$$

If we now insert the formulas for $G, C$ and multiply with $M^{n+3}, \delta=0$ or $M^{n+4}, \delta=1$, we get a polynomial $p$ of degree at most $2(n+3)(\delta=0)$ or $2(n+4) \quad(\delta=1)$ in $M$, such that a positive singular point

$$
\left(C, D, G, M, N, I_{1}, \cdots, I_{n}\right)
$$


will have $p(M)=0$.

Let $\delta=1$. Then we have

Theorem 2. For $\delta=1$, there exist rate constants $k_{i j}>0$, such that (24) holds and all equilibria are positive and stable.

Proof. Now let

$$
\hat{a}=k_{98}-k_{28}
$$

and assume that it is negative. Then

$$
\frac{\partial d_{*}^{C}}{\partial t}(0)=-\frac{k_{82}^{0}}{\hat{a}}
$$

is the same for all treatments. But now the first coordinate of formula (48) becomes

$$
-k_{21} d_{*}^{C} d_{*}^{N}-k_{23} d_{*}^{C} d_{*}^{D}+\hat{a} d_{*}^{C}-2 k_{27} X\left(d_{*}^{C}\right)^{2}+k_{82}-\sum_{i=1}^{n} k_{2,10+2 i} d_{*}^{C} d_{*}^{I_{i}}=0
$$

So

$$
\begin{aligned}
\hat{a} \frac{\partial^{2} d_{*}^{C}}{\partial t^{2}}(0)= & k_{21}\left(\frac{\partial^{2} d_{*}^{C}}{\partial t^{2}} d_{*}^{N}+2 \frac{\partial d_{*}^{C}}{\partial t} \frac{\partial d_{*}^{N}}{\partial t}+d_{*}^{C} \frac{\partial^{2} d_{*}^{N}}{\partial t}\right) \\
& +k_{23}\left(\frac{\partial^{2} d_{*}^{C}}{\partial t^{2}} d_{*}^{D}+2 \frac{\partial d_{*}^{C}}{\partial t} \frac{\partial d_{*}^{D}}{\partial t}+d_{*}^{C} \frac{\partial^{2} d_{*}^{D}}{\partial t}\right) \\
& +\sum_{i=1}^{n} k_{2,10+2 i}\left(\frac{\partial^{2} d_{*}^{C}}{\partial t^{2}} d_{*}^{I_{i}}+2 \frac{\partial d_{*}^{C}}{\partial t} \frac{\partial d_{*}^{I_{i}}}{\partial t}+d_{*}^{C} \frac{\partial^{2} d_{*}^{I_{i}}}{\partial t}\right) \\
& +4 k_{27} X\left(\frac{\partial d_{*}^{C}}{\partial t}\right)^{2}
\end{aligned}
$$

and when $k_{2,10+2 i}=k_{2,11+2 i}=k_{11+2 i, 2}=0, i=1, \cdots, n$.

$$
\begin{aligned}
\hat{a} \frac{\partial^{3} d_{*}^{C}}{\partial t^{3}}(0)= & 3 k_{21}\left(\frac{\partial^{2} d_{*}^{C}}{\partial t^{2}} \frac{\partial d_{*}^{N}}{\partial t}+\frac{\partial d_{*}^{C}}{\partial t} \frac{\partial^{2} d_{*}^{N}}{\partial t^{2}}\right) \\
& +3 k_{23}\left(\frac{\partial^{2} d_{*}^{C}}{\partial t^{2}} \frac{\partial d_{*}^{D}}{\partial t}+\frac{\partial d_{*}^{C}}{\partial t} \frac{\partial^{2} d_{*}^{D}}{\partial t^{2}}\right) \\
& +12 k_{27} X \frac{\partial^{2} d_{*}^{C}}{\partial t^{2}} \frac{\partial d_{*}^{C}}{\partial t}
\end{aligned}
$$

We can now take $k_{21}$ big and argue as in theorem 1 .

\section{The Chemo Radiation Model}

Consider the mass action kinetic system

$$
\begin{aligned}
& C+D \rightarrow 0 \\
& (1+\delta) C+X \rightarrow 0, \delta=0,1 \\
& C \leftrightarrows 2 C \\
& C \leftrightarrows 0 \\
& D \leftrightarrows 0
\end{aligned}
$$


Here the complexes are $C(2)=0, C(3)=C+D, \quad C(7)=(1+\delta) C+X$, $C(8)=C, C(9)=2 C, C(10)=D$. With mass action kinetics the vector field is

$$
f(C, D)=\left(\begin{array}{c}
k_{82}-k_{23} C \cdot D+\left(k_{98}-k_{28}-k_{27} X(1+\delta(2 C-1))\right) C \\
-k_{23} C \cdot D+k_{10,2}-k_{2,10} D
\end{array}\right)
$$

all $k_{i j}>0$.

This system with $\delta=0$ is similar to the reduced system from [17].

$$
\begin{aligned}
& G F \rightarrow C \\
& C+G I \rightarrow 0 \\
& C \rightarrow 2 C \\
& G F \leftrightarrows 0 \\
& G I \leftrightarrows 0 \\
& C \leftrightarrows 0
\end{aligned}
$$

where we have added the last reaction. The complexes are $C(1)=G F$, $C(2)=C, \quad C(3)=C+G I, \quad C(4)=0, \quad C(5)=2 C, \quad C(6)=G I . \quad$ The vector field is

$$
g(C, G F, G I)=\left(\begin{array}{c}
k_{21} G F-k_{43} C \cdot G I+\left(k_{52}-k_{42}\right) C+k_{24} \\
k_{14}-\left(k_{21}+k_{41}\right) G F \\
-k_{43} C \cdot G I+k_{64}-k_{46} G I
\end{array}\right)
$$

We can assume that $G F$ is at equilibrium

$$
G F=\frac{k_{14}}{k_{21}+k_{41}}
$$

The reduced system is then

$$
\begin{aligned}
& \hat{g}(C, G I)=\left(\begin{array}{c}
k-k_{43} C \cdot G I+\left(k_{52}-k_{42}\right) C \\
-k_{43} C \cdot G I+k_{64}-k_{46} G I
\end{array}\right) \\
& k=\frac{k_{21} k_{14}}{k_{21}+k_{41}}+k_{24}
\end{aligned}
$$

We shall find the singular points of the chemo radiation system. $D^{\prime}=0$ gives

$$
D=\frac{k_{10,2}}{k_{23} C+k_{2,10}}
$$

when $C>0$ and then $C^{\prime}=0$ amounts to

$$
k_{82}-k_{23} C \frac{k_{10,2}}{k_{23} C+k_{2,10}}+\left(k_{98}-k_{28}-k_{27} X(1+\delta(2 C-1))\right) C=0
$$

which is equivalent to

$$
\begin{aligned}
& k_{23}\left(k_{98}-k_{28}-k_{27} X\right) C^{2} \\
& +\left(k_{2,10}\left(k_{98}-k_{28}-k_{27} X\right)+k_{23} k_{82}-k_{23} k_{10,2}\right) C+k_{82} k_{2,10}=0
\end{aligned}
$$

when $\delta=0, C>0$ and when $\delta=1, C>0$ it is equivalent to 


$$
\begin{aligned}
& -2 k_{23} k_{27} X C^{3}+\left(\left(k_{98}-k_{28}\right) k_{23}-2 k_{27} X k_{2,10}\right) C^{2} \\
& +\left(k_{2,10}\left(k_{98}-k_{28}\right)+k_{82} k_{23}-k_{23} k_{10,2}\right) C+k_{82} k_{2,10}=0
\end{aligned}
$$

The linearization of $f$ at a singular point $C_{*}=(C, D)$ is

$$
A=D f_{c_{*}}=\left(\begin{array}{cc}
-k_{23} D+\left(k_{98}-k_{28}-k_{27} X\right) & -k_{23} C \\
-k_{23} D & -k_{2,10}-k_{23} C
\end{array}\right)
$$

when $\delta=0$ and when $\delta=1$ it is

$$
\tilde{A}=D f_{c_{*}}=\left(\begin{array}{cc}
-k_{23} D+\left(k_{98}-k_{28}-4 k_{27} X C\right) & -k_{23} C \\
-k_{23} D & -k_{2,10}-k_{23} C
\end{array}\right)
$$

If $\delta=0$

$$
\hat{a}=k_{98}-k_{28}-k_{27} X<0
$$

and $(C, D)$ is a positive singular point, then

$$
\sigma=\operatorname{trace} A<0 \quad \operatorname{det} A=-\hat{a} k_{23} C+k_{2,10}\left(k_{23} D-\hat{a}\right)>0
$$

hence this is a stable, positive singular point.

Consider the chemo radiation system where

$$
k_{10,2}=23 \quad k_{98}=13 \quad X=1 \quad \delta=1
$$

and all other $k_{i j}=1$. Then the cubic polynomial giving candidates of singular points is

$$
-2 C^{3}+10 C^{2}-10 C+1=0
$$

and this gives three singular points

$$
(C, D)=\left\{\begin{array}{l}
(0.112,20.68) \\
(1.21,10.41) \\
(3.677,4.918)
\end{array}\right.
$$

where we have used that

$$
D=\frac{k_{10,2}}{k_{23} C+k_{2,10}}
$$

It is a simple matter to check that the first and last of these are stable equilibria and the middle is an unstable saddle by computing the trace and determinant of the linearization of $f_{q}$ in the singular points. I have plotted a phase portrait for these values of the parameters in Figure 3.

\section{The Quadrapeutics Equilibrium Equation}

We shall find the quadrapeutics equilibrium equation too.

Theorem 3. Suppose $\delta=0$. There exists a polynomial of degree atmost six

$$
s(M)=\sum_{i=0}^{6} q_{i} M^{i} \quad q_{i} \in \mathbb{R}
$$

such that if $(C, D, G, M, N)$ is a positive singular point of $f_{q}$ then

$$
s(M)=0
$$




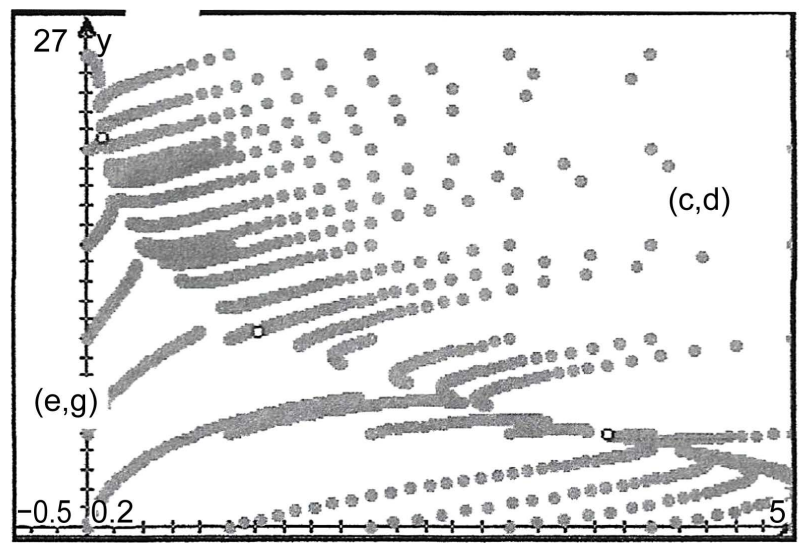

Figure 3. A tristable chemo radiation system. There are three singular points marked with a circle.

Proof. Isolate $D, G, M, N$ from (15), (16), (17), (18) and insert it in $f_{q 1}(C, D, G, M, N)=0$ to find

$$
\begin{aligned}
& p(C, G, M)=-k_{21} C k_{65} M\left(k_{23} C+k_{54} G+k_{2,10}\right) \\
& -k_{23} C k_{10,2}\left(k_{21} C+k_{26}\right) \\
& +\left(k_{82}+\left(k_{98}-k_{28}-k_{27} X(1+\delta(2 C-1))\right) C\right) \\
& \cdot\left(k_{21} C+k_{26}\right)\left(k_{23} C+k_{54} G+k_{2,10}\right)=0
\end{aligned}
$$

where we have multiplied with

$$
\left(k_{21} C+k_{26}\right)\left(k_{23} C+k_{54} G+k_{2,10}\right)
$$

We shall now insert

$$
C=\frac{a M^{2}+b M+c}{M} \quad G=\alpha M+\beta
$$

where

$$
\begin{gathered}
\alpha=-\frac{k_{65}+k_{25}}{k_{2,11}} \\
\beta=\frac{k_{11,2}}{k_{2,11}}
\end{gathered}
$$

in the polynomial $p$. Compute

$$
M^{3} C^{3}=\sum_{i=0}^{6} \tilde{a}_{i} M^{i}
$$

where

$$
\begin{aligned}
& \tilde{a}_{6}=a^{3} \\
& \tilde{a}_{5}=3 a^{2} b \\
& \tilde{a}_{4}=3\left(a b^{2}+a^{2} c\right) \\
& \tilde{a}_{3}=6 a b c+b^{3}
\end{aligned}
$$




$$
\begin{aligned}
& \tilde{a}_{2}=3\left(a c^{2}+b^{2} c\right) \\
& \tilde{a}_{1}=3 b c^{2} \\
& \tilde{a}_{0}=c^{3}
\end{aligned}
$$

Now define

$$
p_{1}(C, G, M)=M^{3}\left(-k_{21} C k_{65} M\left(k_{23} C+k_{54} G+k_{2,10}\right)\right)
$$

and this is

$$
s_{1}(M)=p_{1}\left(\frac{a M^{2}+b M+c}{M}, \alpha M+\beta, M\right)
$$

Also put

$$
p_{2}(C, G, M)=M^{3}\left(-k_{23} C k_{10,2}\left(k_{21} C+k_{26}\right)\right)
$$

and this is

$$
s_{2}(M)=p_{2}\left(\frac{a M^{2}+b M+c}{M}, \alpha M+\beta, M\right)
$$

Finally set

$$
p_{3}(C, G, M)=M^{3}\left(k_{82}+\hat{a} C\right)\left(k_{21} C+k_{26}\right)\left(k_{23} C+k_{54} G+k_{2,10}\right)
$$

which is

$$
s_{3}(M)=p_{3}\left(\frac{a M^{2}+b M+c}{M}, \alpha M+\beta, M\right)
$$

So

$$
s(M)=s_{1}(M)+s_{2}(M)+s_{3}(M)
$$

Now compute

$$
\begin{aligned}
s_{1}(M)= & -M^{2} k_{21} k_{65} k_{23}\left(a^{2} M^{4}+b^{2} M^{2}+c^{2}+2 a b M^{3}+2 a c M^{2}+2 b c M\right) \\
& \left.-k_{21}\left(a M^{2}+b M+c\right) k_{65} M^{3}\left(k_{54}(\alpha M+\beta)\right)+k_{2,10}\right) \\
= & -k_{21} k_{65} k_{23}\left(a^{2} M^{6}+b^{2} M^{4}+c^{2} M^{2}+2 a b M^{5}+2 a c M^{4}+2 b c M^{3}\right) \\
& -k_{21} k_{65}\left(a M^{2}+b M+c\right) M^{3} k_{54} \alpha M \\
& -k_{21} k_{65}\left(a M^{2}+b M+c\right) M^{3}\left(k_{54} \beta+k_{2,10}\right)
\end{aligned}
$$

Write

$$
s_{1}(M)=\sum_{i=0}^{6} a_{i} M^{i}
$$

Then by the previous computation

$$
\begin{aligned}
& a_{6}=-k_{21} a k_{65}\left(k_{23} a+k_{54} \alpha\right) \\
& a_{5}=-k_{21} b k_{65}\left(k_{23} a+k_{54} \alpha\right)-k_{21} a k_{65}\left(k_{54} \beta+k_{23} b+k_{2,10}\right) \\
& a_{4}=-k_{21} c k_{65}\left(k_{23} a+k_{54} \alpha\right)-k_{21} b k_{65}\left(k_{54} \beta+k_{23} b+k_{2,10}\right)-k_{21} k_{65} k_{23} a c
\end{aligned}
$$




$$
\begin{aligned}
& a_{3}=-k_{21} c k_{65}\left(k_{54} \beta+k_{23} b+k_{2,10}\right)-k_{21} k_{65} k_{23} b c \\
& a_{2}=-k_{21} c^{2} k_{65} k_{23} \\
& a_{1}=0 \\
& a_{0}=0
\end{aligned}
$$

Now compute

$$
\begin{aligned}
s_{2}(M)= & M^{3}\left(-k_{23} C k_{10,2}\left(k_{21} C+k_{26}\right)\right) \\
= & -M^{3} k_{23} k_{10,2}\left(k_{21} \frac{a^{2} M^{4}+b^{2} M^{2}+c^{2}+2 a b M^{3}+2 a c M^{2}+2 b c M}{M^{2}}\right. \\
& \left.+k_{26} \frac{a M^{2}+b M+c}{M}\right) \\
= & -k_{23} k_{10,2} k_{21}\left(a^{2} M^{5}+b^{2} M^{3}+c^{2} M+2 a b M^{4}+2 a c M^{3}+2 b c M^{2}\right) \\
& -k_{23} k_{10,2} k_{26}\left(a M^{4}+b M^{3}+c M^{2}\right)
\end{aligned}
$$

Write

$$
S_{2}(M)=\sum_{i=0}^{6} b_{i} M^{i}
$$

and by the computation above

$$
\begin{aligned}
& b_{6}=0 \\
& b_{5}=-k_{23} a^{2} k_{10,2} k_{21} \\
& b_{4}=-k_{23} b k_{10,2} k_{21} a-k_{23} a k_{10,2}\left(k_{21} b+k_{26}\right) \\
& b_{3}=-2 k_{23} k_{10,2} k_{21} a c-k_{23} k_{10,2} b\left(k_{21} b+k_{26}\right) \\
& b_{2}=-k_{23} c k_{10,2}\left(k_{21} b+k_{26}\right)-k_{23} b k_{10,2} k_{21} c \\
& b_{1}=-k_{23} c^{2} k_{10,2} k_{21} \\
& b_{0}=0
\end{aligned}
$$

Now consider

$$
\begin{aligned}
p_{3}(C, G, M)= & k_{21} k_{23} \hat{a} C^{3}+k_{21} k_{54} \hat{a} C^{2} G \\
& +k_{21} \hat{a} C^{2} k_{2,10}+k_{26} k_{23} \hat{a} C^{2} \\
& +k_{26} k_{54} G \hat{a} C+k_{26} k_{2,10} \hat{a} C \\
& +k_{82} k_{21} k_{23} C^{2}+k_{82} k_{21} k_{54} C G \\
& +k_{82} k_{21} k_{2,10} C+k_{82} k_{26} k_{23} C \\
& +k_{82} k_{26} k_{54} G+k_{82} k_{26} k_{2,10}
\end{aligned}
$$

Name these 12 summands $d_{1}, \cdots, d_{12}$. Now

$$
M^{3} d_{1}=k_{21} k_{23} \hat{a} \sum_{i=0}^{6} \tilde{a}_{i} M^{i}
$$

and 


$$
\begin{aligned}
M^{3} d_{2}= & k_{21} k_{54} \hat{a}\left(\alpha\left(a^{2} M^{6}+b^{2} M^{4}+c^{2} M^{2}+2 a b M^{5}+2 a c M^{4}+2 b c M^{3}\right)\right. \\
& \left.+\beta\left(a^{2} M^{5}+b^{2} M^{3}+c^{2} M+2 a b M^{4}+2 a c M^{3}+2 b c M^{2}\right)\right)
\end{aligned}
$$

Also

$$
M^{3} d_{3}=k_{21} \hat{a} k_{2,10}\left(a^{2} M^{5}+b^{2} M^{3}+c^{2} M+2 a b M^{4}+2 a c M^{3}+2 b c M^{2}\right)
$$

Similarly

$$
M^{3} d_{4}=k_{26} \hat{a} k_{23}\left(a^{2} M^{5}+b^{2} M^{3}+c^{2} M+2 a b M^{4}+2 a c M^{3}+2 b c M^{2}\right)
$$

Then

$$
M^{3} d_{5}=k_{26} k_{54} \hat{a}\left(\alpha a M^{5}+\alpha b M^{4}+c \alpha M^{3}+\beta a M^{4}+\beta b M^{3}+\beta c M^{2}\right)
$$

And

$$
M^{3} d_{6}=k_{26} k_{2,10} \hat{a}\left(a M^{4}+b M^{3}+c M^{2}\right)
$$

We also find

$$
\begin{aligned}
& M^{3} d_{7}=k_{82} k_{21} k_{23}\left(a^{2} M^{4}+b^{2} M^{2}+c^{2}+2 a b M^{3}+2 a c M^{2}+2 b c M\right) M \\
& M^{3} d_{8}=k_{82} k_{21} k_{54}\left(\alpha\left(a M^{5}+b M^{4}+c M^{3}\right)+\beta\left(a M^{4}+b M^{3}+c M^{2}\right)\right)
\end{aligned}
$$

Combine

$$
M^{3}\left(d_{9}+d_{10}\right)=\left(k_{82} k_{21} k_{2,10}+k_{82} k_{26} k_{23}\right)\left(a M^{4}+b M^{3}+c M^{2}\right)
$$

The last two $d s$ give

$$
\begin{aligned}
& M^{3} d_{11}=k_{82} k_{26} k_{54}\left(\alpha M^{4}+\beta M^{3}\right) \\
& M^{3} d_{12}=k_{82} k_{26} k_{2,10} M^{3}
\end{aligned}
$$

Write

$$
S_{3}(M)=\sum_{i=0}^{6} c_{i} M^{i}
$$

Then we find from the above computations

$$
c_{6}=\hat{a} a^{2} k_{21}\left(k_{23} a+k_{54} \alpha\right)
$$

and

$$
\begin{aligned}
c_{5}= & k_{21} k_{23} \hat{a} 3 a^{2} b+k_{21} k_{54} \hat{a}\left(\alpha 2 a b+\beta a^{2}\right)+k_{21} \hat{a} k_{2,10} a^{2} \\
& +k_{26} k_{23} \hat{a} a^{2}+k_{82} k_{21} k_{23} a^{2}+k_{82} k_{21} k_{54} a \alpha+k_{26} k_{54} \hat{a} \alpha a
\end{aligned}
$$

Now collect terms

$$
\begin{aligned}
c_{4}= & k_{21} k_{23} \hat{a} 3\left(a b^{2}+a^{2} c\right)+k_{21} k_{54} \hat{a} \alpha\left(b^{2}+2 a c\right) \\
& +k_{21} k_{54} \hat{a} \beta 2 a b+k_{21} \hat{a} k_{2,10} 2 a b+k_{26} k_{23} \hat{a} 2 a b \\
& +k_{26} k_{54} \hat{a}(\alpha b+\beta a)+k_{26} k_{2,10} \hat{a} a \\
& +k_{82} k_{21} k_{23} 2 a b+k_{82} k_{21} k_{54}(\alpha b+\beta a) \\
& +\left(k_{82} k_{21} k_{2,10}+k_{82} k_{26} k_{23}\right) a+k_{82} k_{26} k_{54} \alpha
\end{aligned}
$$


We can also find

$$
\begin{aligned}
c_{3}= & k_{21} k_{23} \hat{a}\left(6 a b c+b^{3}\right)+k_{21} k_{54} \hat{a}\left(2 b c \alpha+\beta\left(b^{2}+2 a c\right)\right) \\
& +k_{21} \hat{a} k_{2,10}\left(b^{2}+2 a c\right)+k_{26} k_{23} \hat{a}\left(b^{2}+2 a c\right) \\
& +k_{26} k_{54} \hat{a}(c \alpha+\beta b)+k_{26} k_{2,10} \hat{a} b \\
& +k_{82} k_{21} k_{23}\left(b^{2}+2 a c\right)+k_{82} k_{21} k_{54}(\alpha c+\beta b) \\
& +\left(k_{82} k_{21} k_{2,10}+k_{82} k_{26} k_{23}\right) b+k_{82} k_{26} k_{54} \beta+k_{82} k_{26} k_{2,10}
\end{aligned}
$$

Now

$$
\begin{aligned}
c_{2}= & k_{21} k_{23} \hat{a} 3\left(a c^{2}+b^{2} c\right)+k_{21} k_{54} \hat{a}\left(\alpha c^{2}+\beta 2 b c\right) \\
& +\left(k_{21} \hat{a} k_{2,10}+k_{26} k_{23} \hat{a}\right) 2 b c+k_{26} k_{54} \hat{a} \beta c \\
& +k_{26} k_{2,10} \hat{a} c+k_{82} k_{21} k_{23} 2 b c \\
& +k_{82} k_{21} k_{54} \beta c+\left(k_{82} k_{21} k_{2,10}+k_{82} k_{26} k_{23}\right) c
\end{aligned}
$$

Also

$$
\begin{aligned}
c_{1}= & k_{21} k_{23} \hat{a} 3 b c^{2}+k_{21} k_{54} \hat{a} c^{2} \beta+k_{21} \hat{a} k_{2,10} c^{2} \\
& +k_{82} k_{21} k_{23} c^{2}+k_{23} c^{2} \hat{a} k_{26}
\end{aligned}
$$

Finally

$$
c_{0}=k_{21} k_{23} \hat{a} c^{3}
$$

The quadrapeutics polynomial is

$$
s(M)=\sum_{i=0}^{6}\left(a_{i}+b_{i}+c_{i}\right) M^{i}
$$

If all rate constants $k_{i j}=1, X=1$ then

$$
s(M)=2 M^{5}-8 M^{4}+10 M^{3}-\frac{23}{4} M^{2}+\frac{3}{2} M-\frac{1}{8}=0
$$

This polynomium has three real roots

$$
M=0.148638, M=\frac{1}{2}, M=2.32234
$$

and two imaginary roots

$$
0.514511 \pm 0.312089 i
$$

There are thus two candidates of singular points $\left(M=\frac{1}{2}\right.$ does not give a singular point).

$$
(C, D, G, M, N)=\left\{\begin{array}{l}
(0.661153,0.423634,0.702724,0.148638,0.089479) \\
(1.85998,-1.27437,-3.64468,2.32234,0.812013)
\end{array}\right.
$$

and they are both singular points (numerical evidence). We have used, that 


$$
\begin{aligned}
& G=\alpha M+\beta \\
& C=\frac{a M^{2}+b M+c}{M} \\
& D=\frac{k_{10,2}}{k_{23} C+k_{54} G+k_{2,10}} \\
& N=\frac{k_{65} M}{k_{21} C+k_{26}}
\end{aligned}
$$

\section{The Extended Quadrapeutics Model}

This is the system (1) to (11) and the reactions

$$
\begin{aligned}
& \tilde{D}+C \rightarrow 0 \\
& N+D \rightarrow \tilde{D} \\
& \tilde{D} \rightarrow 0 \\
& D \rightarrow \tilde{D}
\end{aligned}
$$

taking into account, that plasmonic nanobubbles destroy the liposomes $D$ and thus the chemo therapeutic drug $\tilde{D}$ is injected into the cytoplasm. But some of the liposomes decay, producing chemo therapeutic drug. The complexes here are $C(12)=N+D, C(13)=\tilde{D}, C(14)=\tilde{D}+C$. The ODEs are

$$
\begin{aligned}
& C^{\prime}=-k_{21} C \cdot N-k_{23} C \cdot D+\left(k_{98}-k_{28}-k_{27} X\right) C+k_{82}-k_{2,14} \tilde{D} \cdot C \\
& \tilde{D}^{\prime}=-k_{2,14} \tilde{D} \cdot C+k_{13,12} N \cdot D-k_{2,13} \tilde{D}+k_{13,10} D \\
& D^{\prime}=-k_{23} C \cdot D-k_{54} D \cdot G+k_{10,2}-k_{2,10} D-k_{13,12} N \cdot D-k_{13,10} D \\
& G^{\prime}=-k_{54} D \cdot G+k_{11,2}-k_{2,11} G \\
& M^{\prime}=k_{54} D \cdot G-\left(k_{65}+k_{25}\right) M \\
& N^{\prime}=-k_{21} C \cdot N+k_{65} M-k_{26} N-k_{13,12} N \cdot D
\end{aligned}
$$

The vector field is denoted $g_{q}$. Denote by $g_{c r}$ the vector field in (260), (261) and (262) with the rate constants in (263), (264) and (265) all equal to zero and the rest positive.

Theorem 4. There exist positive values of the rate constants such that

$$
C_{*}^{q}<C_{*}^{c r}
$$

holds and the singular points are both positive and stable.

Proof. Define for $*=q$

$$
\begin{aligned}
& k_{1}=\left(k_{82}, k_{10,2}, k_{11,2}\right) \\
& k_{2}=\left(k_{21}, k_{23}, k_{98}, k_{28}, \tilde{k}_{27}, k_{2,14}, k_{13,12}, k_{2,13}, k_{54}, k_{2,10}, k_{2,11}, k_{65}, k_{25}, k_{26}, k_{13,10}\right) \\
& \text { And for * } * \text { cr } \\
& \qquad k_{1}^{c r}=\left(k_{82}, k_{10,2}\right) \\
& \qquad k_{2}^{c r}=\left(k_{23}, k_{98}, k_{28}, \tilde{k}_{27}, k_{2,14}, k_{2,13}, k_{2,10}, k_{13,10}\right)
\end{aligned}
$$


Define

$$
K_{c r}=\left(k_{1}^{c r}, k_{2}^{c r}\right) \quad K_{q}=\left(k_{1}^{q}, k_{2}^{q}\right)
$$

and

$$
\hat{K}_{*}=\left(k_{1}^{*}, k_{2}^{*}\right) \quad k_{1}^{*}=0
$$

Use the implicit function theorem to find a mapping

$$
c_{q}: V_{q} \subset \mathbb{R}^{18} \rightarrow \mathbb{R}^{6}
$$

and

$$
c_{c r}: V_{c r} \subset \mathbb{R}^{10} \rightarrow \mathbb{R}^{3}
$$

such that

$$
g_{*}\left(c_{*}\left(K_{*}\right), K_{*}\right)=0 \quad C_{*}\left(\hat{K}_{*}\right)=0
$$

and define

$$
d_{*}(t)=c_{*}\left(t k_{1}^{*, 0}, k_{2}^{*}\right) *=\left\{\begin{array}{l}
c r \\
q
\end{array}\right.
$$

$V_{q}, V_{c r}$ are open subsets of $\mathbb{R}^{18}, \mathbb{R}^{10}$ respectively. For $*=c r$ let $d_{*}^{G}=d_{*}^{M}$ $=d_{*}^{N}=0$. By the implicit function theorem

$$
d_{*}^{\tilde{D}}(0)=0
$$

Differentiate (262) and (261) with respect to $t$ to get

$$
\frac{\partial d_{*}^{D}}{\partial t}=\frac{k_{10,2}^{0}}{k_{2,10}+k_{13,10}}
$$

and

$$
\frac{\partial d_{*}^{\tilde{D}}}{\partial t}=\frac{k_{10,2}^{0} k_{13,10}}{k_{2,13}\left(k_{2,10}+k_{13,10}\right)}
$$

Now differentiate (261) twice with respect to $t$ to find

$$
k_{2,13} \frac{\partial^{2} d_{*}^{\tilde{D}}}{\partial t^{2}}=k_{13,10} \frac{\partial^{2} d_{*}^{D}}{\partial t^{2}}-2 k_{2,14} \frac{\partial d_{*}^{\tilde{D}}}{\partial t} \frac{\partial d_{*}^{C}}{\partial t}
$$

and differentiate (262) with respect to $t$ to get

$$
\frac{\partial^{2} d_{*}^{D}}{\partial t^{2}}=-\frac{2}{k_{2,10}+k_{13,10}}\left(k_{23} \frac{\partial d_{*}^{C}}{\partial t} \frac{\partial d_{*}^{D}}{\partial t}+k_{54} \frac{\partial d_{*}^{D}}{\partial t} \frac{\partial d_{*}^{G}}{\partial t}\right)
$$

We also have

$$
\frac{\partial d_{*}^{C}}{\partial t}=-\frac{k_{82}^{0}}{\hat{a}} \quad \frac{\partial d_{*}^{G}}{\partial t}=\frac{k_{11,2}^{0}}{k_{2,11}}
$$

the last equality when $*=q$. We now get

$$
\frac{\partial^{2} d_{*}^{C}}{\partial t^{2}}=\frac{1}{\hat{a}}\left(2 k_{23} \frac{\partial d_{*}^{C}}{\partial t} \frac{\partial d_{*}^{D}}{\partial t}+2 k_{2,14} \frac{\partial d_{*}^{\tilde{D}}}{\partial t} \frac{\partial d_{*}^{C}}{\partial t}+k_{21} \frac{\partial d_{*}^{C}}{\partial t} \frac{\partial d_{*}^{N}}{\partial t}\right)
$$


so

$$
\frac{\partial^{2} d_{c r}^{C}}{\partial t^{2}}(0)=\frac{\partial^{2} d_{q}^{C}}{\partial t^{2}}(0)
$$

and

$$
\frac{\partial^{2} d_{*}^{N}}{\partial t^{2}}=\frac{k_{65} 2 k_{54}}{k_{26}\left(k_{65}+k_{25}\right)} \frac{\partial d_{*}^{D}}{\partial t} \frac{\partial d_{*}^{G}}{\partial t}
$$

where we have used that

$$
\frac{\partial d_{*}^{M}}{\partial t}(0)=0
$$

hence

$$
\frac{\partial d_{*}^{N}}{\partial t}(0)=0
$$

Finally

$$
\begin{aligned}
\hat{a} \frac{\partial^{3} d_{*}^{C}}{\partial t^{3}}= & 3 k_{23}\left(\frac{\partial^{2} d_{*}^{C}}{\partial t^{2}} \frac{\partial d_{*}^{D}}{\partial t}+\frac{\partial d_{*}^{C}}{\partial t} \frac{\partial^{2} d_{*}^{D}}{\partial t^{2}}\right) \\
& +3 k_{2,14}\left(\frac{\partial^{2} d_{*}^{\tilde{D}}}{\partial t^{2}} \frac{\partial d_{*}^{C}}{\partial t}+\frac{\partial d_{*}^{\tilde{D}}}{\partial t} \frac{\partial^{2} d_{*}^{C}}{\partial t^{2}}\right) \\
& +3 k_{21} \frac{\partial d_{*}^{C}}{\partial t} \frac{\partial^{2} d_{*}^{N}}{\partial t^{2}}
\end{aligned}
$$

and taking $k_{21}$ big, this implies the theorem, arguing as in the proof of theorem 1 .

From $D^{\prime}=0$ isolate

$$
C=\frac{1}{k_{23} D}\left(-k_{54} D \cdot G+k_{10,2}-\left(k_{2,10}+k_{13,10}\right) D-k_{13,12} N \cdot D\right)
$$

and insert this in $N^{\prime}=0$ to get using

$$
G=\alpha M+\beta \quad \alpha<0 \quad \beta>0
$$

and

$$
D=\frac{-\alpha k_{2,11} M}{k_{54}(\alpha M+\beta)}
$$

(this equation follows from (263)), that

$$
\begin{aligned}
& \frac{k_{13,12} k_{21}}{k_{23}} N^{2}+N\left(k_{54} \frac{k_{21}}{k_{23}}(\alpha M+\beta)+\frac{k_{21} k_{10,2} k_{54}(\alpha M+\beta)}{k_{23} k_{2,11} \alpha M}\right. \\
& \left.+k_{21} \frac{k_{2,10}+k_{13,10}}{k_{23}}-k_{26}+k_{13,12} \frac{k_{2,11} \alpha M}{k_{54}(\alpha M+\beta)}\right)+k_{65} M=0
\end{aligned}
$$

$M>0, M \neq-\frac{\beta}{\alpha}$. Let $\tilde{a}$ be the coefficient to $N^{2}$ and let $\tilde{b}(M)$ be the coefficient to $N$ and $\tilde{c}(M)=k_{65} M$. Then we get when 


$$
\begin{gathered}
\Delta=\tilde{b}(M)^{2}-4 \tilde{a} \tilde{c}(M)>0 \\
N^{ \pm}(M)=\frac{-\tilde{b}(M) \pm \sqrt{\Delta}}{2 \tilde{a}}
\end{gathered}
$$

$\tilde{a}>0$. Thus $D^{\prime}=0$ gives

$$
C^{ \pm}=\frac{1}{k_{23} D(M)}\left(-k_{54} D \cdot G+k_{10,2}-\left(k_{2,10}+k_{13,10}\right) D-k_{13,12} N^{ \pm} \cdot D\right)
$$

and

$$
\begin{aligned}
& \tilde{D}^{ \pm}=\frac{k_{13,12} N^{ \pm} D+k_{13,10} D}{k_{2,14} C^{ \pm}+k_{2,13}} \\
& D=\frac{-k_{2,11} \alpha M}{k_{54}(\alpha M+\beta)}
\end{aligned}
$$

So let

$$
p_{*}(M)=p_{*}^{ \pm}(M)=-k_{21} C^{ \pm} N^{ \pm}-k_{23} C^{ \pm} D+\hat{a} C^{ \pm}+k_{82}-k_{2,14} \tilde{D}^{ \pm} C^{ \pm}
$$

If $(C, \tilde{D}, D, G, M, N)$ is a positive singular point of $g_{q}$, then

$$
p_{*}^{ \pm}(M)=0
$$

Define $h$ by

$$
\begin{aligned}
\sqrt{1+x}-1 & =\int_{0}^{1} \frac{\partial}{\partial s} \sqrt{1+x s} \mathrm{~d} s \\
& =x \int_{0}^{1} \frac{1}{2 \sqrt{1+x s}} \mathrm{~d} s=x h(x)
\end{aligned}
$$

where $h$ is smooth and $x \in]-1,+\infty[$.

Proposition 5. The function

$$
N^{-}(M) \triangleq-2 \frac{\tilde{c}(M)}{\tilde{b}(M)} h\left(-\frac{4 \tilde{a} \tilde{c}}{\tilde{b}^{2}}\right)
$$

satisfies (294) and (295), when

$$
\left.-\frac{4 \tilde{a} \tilde{c}}{\tilde{b}^{2}} \in\right]-1,+\infty[, \quad \tilde{b}(M)<0
$$

Proof. If $\tilde{b}(M)<0$ then

$$
N^{-}(M)=-\frac{2 \tilde{c}(M)}{\tilde{b}(M)} h\left(-\frac{4 \tilde{a} \tilde{c}(M)}{\tilde{b}(M)^{2}}\right)
$$

is smooth and

$$
N^{+}(M)=\frac{-\tilde{b}(M)+\sqrt{\Delta(M)}}{2 \tilde{a}}
$$

when $\tilde{a}>0$. If $\tilde{b}(M)>0$ then

$$
N^{+}(M)=-\frac{2 \tilde{c}(M)}{\tilde{b}(M)} h\left(-\frac{4 \tilde{a} \tilde{c}(M)}{\tilde{b}(M)^{2}}\right)
$$


is smooth and

$$
N^{-}(M)=\frac{-\tilde{b}(M)-\sqrt{\Delta(M)}}{2 \tilde{a}}
$$

when $\tilde{a}>0$. These are both negative, so $\tilde{b}(M)>0$ is not interesting.

Proposition 6. If $k_{13,12}=k_{2,13}=k_{2,14}=k_{13,10}=0$ and (306) holds, then

$$
s(M)=q(M) p_{*}^{-}(M)
$$

where

$$
q(M)=M^{3}\left(k_{21} C+k_{26}\right)\left(k_{23} C+k_{54} G+k_{2,10}\right)
$$

and

$$
\begin{aligned}
& C=\frac{a M^{2}+b M+c}{M} \\
& G=\alpha M+\beta
\end{aligned}
$$

Proof. We have

$$
\begin{aligned}
& k_{23} C+k_{54} G+k_{2,10} \\
& =k_{23} \frac{a M^{2}+b M+c}{M}+k_{54}(\alpha M+\beta)+k_{2,10}
\end{aligned}
$$

and here the terms with $a, \alpha$ cancel out to give

$$
\begin{aligned}
& k_{23} b+k_{54} \beta+k_{2,10}+k_{23} \frac{C}{M} \\
& =-\frac{k_{54} k_{10,2}}{k_{2,11}}+k_{23} \frac{C}{M}
\end{aligned}
$$

So

$$
\begin{gathered}
\frac{k_{10,2}}{k_{23} C+k_{54} G+k_{2,10}} \\
=\frac{k_{10,2}}{-\frac{k_{54} k_{10,2}}{k_{2,11}}+k_{23} \frac{c}{M}}
\end{gathered}
$$

which becomes

$$
\begin{aligned}
& \frac{\left(k_{65}+k_{25}\right) M}{\frac{k_{54} k_{11,2}}{k_{2,11}}-k_{54} \frac{k_{65}+k_{25}}{k_{2,11}} M} \\
& =\frac{-k_{2,11} \alpha M}{k_{54}(\alpha M+\beta)}
\end{aligned}
$$

We also can compute

$$
\begin{aligned}
\tilde{b}(M)= & k_{54} \frac{k_{21}}{k_{23}}(\alpha M+\beta)+\frac{k_{21} k_{10,2} k_{54}}{k_{23} k_{2,11}} \frac{\alpha M+\beta}{\alpha M} \\
& +k_{21} \frac{k_{2,10}}{k_{23}}-k_{26}
\end{aligned}
$$




$$
\begin{aligned}
= & k_{54} \frac{k_{21}}{k_{23}}\left(-\frac{k_{65}+k_{25}}{k_{2,11}} M+\frac{k_{11,2}}{k_{2,11}}\right) \\
& +k_{21} \frac{k_{2,10}}{k_{23}}+\frac{k_{21} k_{10,2} k_{54}}{k_{23} k_{2,11}}-\frac{k_{21} k_{10,2} k_{54}}{M k_{23} k_{2,11}} \frac{k_{11,2}}{k_{65}+k_{25}}-k_{26} \\
= & -k_{21} \frac{a M^{2}+b M+c}{M}-k_{26}=-k_{21} C-k_{26}
\end{aligned}
$$

Notice that

$$
N^{-}(M)=-\frac{\tilde{c}(M)}{\tilde{b}(M)}=\frac{\tilde{c}(M)}{k_{21} C+k_{26}}
$$

and

$$
D=\frac{k_{10,2}}{k_{23} C+k_{54} G+k_{2,10}}
$$

Now we get

$$
\begin{aligned}
p_{*}^{-}(M) q(M) & =\left(-k_{21} C \cdot N^{-}-k_{23} C \cdot D+\left(k_{82}+\hat{a} C\right)\right) q(M) \\
& =-k_{65} M \cdot C\left(k_{23} C+k_{54} G+k_{2,10}\right) M^{3} \\
-k_{23} C\left(k_{21} C+\right. & \left.k_{26}\right) M^{3}+s_{3}(M)=s_{1}(M)+s_{2}(M)+s_{3}(M)=s(M)
\end{aligned}
$$

If we assume there exists $M_{i}>0$ such that

$$
\begin{aligned}
& s\left(M_{i}\right)=0 \\
& \frac{\partial s}{\partial M}\left(M_{i}\right) \neq 0
\end{aligned}
$$

and $q\left(M_{i}\right)>0$ (this is so if $G\left(M_{i}\right), C\left(M_{i}\right)>0$ ). Then

$$
\begin{aligned}
& p_{*}^{-}\left(M_{i}, k_{0}\right)=0 \\
& \frac{\partial p_{*}}{\partial M}\left(M_{i}, k_{0}\right) \neq 0
\end{aligned}
$$

where

$$
k_{0}=K_{q \mid k_{13,12}=k_{13,10}=k_{2,13}=k_{2,14}=k_{13,10}=0}
$$

Then there exists a $C^{1}$ function

$$
M_{*}^{i}: U \rightarrow \mathbb{R}
$$

such that

$$
\begin{aligned}
& p_{*}^{-}\left(M_{*}^{i}(k), k\right)=0 \quad k \in U \\
& M_{*}^{i}\left(k_{0}\right)=M_{i}
\end{aligned}
$$

by the implicit function theorem. Here $U$ is an open neighbourhood of $k_{0}$ in $\mathbb{R}^{18}$. So the roots of $s$ are nearly zeroes of $p_{*}^{-}$, when

$$
k_{2,14}, k_{13,12}, k_{2,13}, k_{13,10}
$$


are small and (333) and (334) holds. The example in the end of section 4 applies here.

\section{Summary}

Consider the extended quadrapeutics system and the additional reactions

$$
\begin{aligned}
& L_{a}+C \rightarrow 0 \\
& L_{a} \leftrightarrows 0
\end{aligned}
$$

Here the complexes are $C(15)=L_{a}+C, C(16)=L_{a}$, modelling adoptive $\mathrm{T}$ cell therapy, see [4]. $L_{a}$ are activated and expanded tumor infiltrating lymphocytes. They are injected back into the patient. Then the differential equations are

$$
\begin{aligned}
& \frac{\mathrm{d} C}{\mathrm{~d} t}=-k_{21} C \cdot N-k_{23} C \cdot D+\hat{a} C+k_{82}-k_{2,14} \tilde{D} \cdot C-k_{2,15} L_{a} \cdot C \\
& \tilde{D}^{\prime}=-k_{2,14} \tilde{D} \cdot C+k_{13,12} N \cdot D-k_{2,13} \tilde{D}+k_{13,10} D \\
& D^{\prime}=-k_{23} C \cdot D-k_{54} D \cdot G+k_{10,2}-k_{2,10} D-k_{13,12} N \cdot D-k_{13,10} D \\
& G^{\prime}=-k_{54} D \cdot G+k_{11,2}-k_{2,11} G \\
& M^{\prime}=k_{54} D \cdot G-\left(k_{65}+k_{25}\right) M \\
& N^{\prime}=-k_{21} C \cdot N+k_{65} M-k_{26} N-k_{13,12} N \cdot D \\
& \frac{\mathrm{d} L_{a}}{\mathrm{~d} t}=-k_{2,15} L_{a} \cdot C+k_{16,2}-k_{2,16} L_{a}
\end{aligned}
$$

Denote this vector field $g_{q}$ and let $g_{c r}$ denote the vector field in (344), (345), (346) where the rate constants in (347), (348), (349), (350) are all zero and the rest positive. Use the implicit function theorem to find maps

$$
c_{c r}: V_{c r} \subset \mathbb{R}^{10} \rightarrow \mathbb{R}^{3}
$$

and

$$
c_{q}: V_{q} \subset \mathbb{R}^{21} \rightarrow \mathbb{R}^{7}
$$

such that

$$
g_{*}\left(c_{*}\left(K_{*}\right), K_{*}\right)=0 \quad c_{*}\left(\hat{K}_{*}\right)=0
$$

Here

$$
K_{c r}=\left(k_{1}^{c r}, k_{2}^{c r}\right)
$$

is the same as in section 5. Also $V_{c r}, V_{q}$ are open subsets of $\mathbb{R}^{10}$ and $\mathbb{R}^{21}$ respectively. And

$$
k_{1}^{q}=\left(k_{82}, k_{10,2}, k_{11,2}, k_{16,2}\right)
$$

Also

$$
\begin{aligned}
k_{2}^{q}=( & k_{21}, k_{23}, k_{98}, k_{28}, \tilde{k}_{27}, k_{2,14}, k_{13,12}, k_{2,13}, k_{54}, k_{2,10}, k_{2,11}, k_{65}, k_{25}, \\
& \left.k_{26}, k_{2,15}, k_{2,16}, k_{13,10}\right)
\end{aligned}
$$

Finally set 


$$
K_{*}=\left(k_{1}^{*}, k_{2}^{*}\right) \quad \hat{K}_{*}=\left(k_{1}^{*}, k_{2}^{*}\right) \quad k_{1}^{*}=0
$$

Define

$$
d_{*}(t)=c_{*}\left(t k_{1}^{*, 0}, k_{2}^{*}\right)
$$

Let

$$
\hat{a}=k_{98}-k_{28}-k_{27} X
$$

and assume that it is negative. Then

$$
\hat{a} \frac{\partial^{2} d_{*}^{C}}{\partial t^{2}}=2 k_{23} \frac{\partial d_{*}^{C}}{\partial t} \frac{\partial d_{*}^{D}}{\partial t}+2 k_{2,15} \frac{\partial d_{*}^{L_{a}}}{\partial t} \frac{\partial d_{*}^{C}}{\partial t}+2 k_{2,14} \frac{\partial d_{*}^{\tilde{D}}}{\partial t} \frac{\partial d_{*}^{C}}{\partial t}
$$

But

$$
\begin{aligned}
\frac{\partial d_{*}^{C}}{\partial t} & =-\frac{k_{82}^{0}}{\hat{a}} \\
\frac{\partial d_{*}^{D}}{\partial t} & =\frac{k_{10,2}^{0}}{k_{2,10}} \\
\frac{\partial d_{*}^{L_{a}}}{\partial t} & =\frac{k_{16,2}^{0}}{k_{2,16}} \\
\frac{\partial d_{*}^{\tilde{D}}}{\partial t} & =\frac{k_{10,2}^{0} k_{13,10}}{k_{2,13}\left(k_{2,10}+k_{13,10}\right)}
\end{aligned}
$$

suggesting, that in this model multipeutics can be more potent than quadrapeutics.

In the present paper, we considered quadrapeutics and multipeutics cancer therapies. We proved the multipeutics theorem, stating that the more treatments we apply the smaller the cancer burden. We also found a polynomial of degree at most 6 , giving candidates of singular points for the quadrapeutics system.

The cells of the immune system have a plasma membrane repair system and it turns out that this system is much more efficient in cancer cells than in normal cells. The two proteins S100A11 and Annexin A2 are involved in this plasma membrane repair system. They are commonly upregulated in cancer cells, see [20]. So we can ask: can cancer cells repair nanobubble injury.

\section{References}

[1] Lukianova-Hleb, E.Y., Kim, Y.S., Aryasomayajula, B., Boulikas, T., Phan, J., Hung, M.C., Torchillin, V.P., O’Neill, B.E. and Lapotko, D.O. (2015) Safety and Efficacy of Quadrapeutics versus Chemoradiation in Head and Neck Carcinoma Xenograft Model. American Journal of Cancer Research, 5, 3534-3547.

[2] Larsen, J.C. (2017) Models of Cancer Growth. Journal of Applied Mathematics and Computing, 53, 613-645.

[3] Larsen, J.C. (2016) Hopf Bifurcations in Cancer Models. JP Journal of Applied Mathematics, 14, 1-31.

[4] Larsen, J.C. (2016) A Mathematical Model of Adoptive T Cell Therapy. JP Journal of Applied Mathematics, to Appear.

[5] Larsen, J.C. (2016) Fundamental Concepts in Dynamics. Survey Article. 
[6] Adam, J.A. and Bellomo, N. (1997) A Survey of Models for Tumor-Induced Immune System Dynamics. Birkhäuser, Boston.

[7] Geha, R. and Notarangelo, L. (2012) Case Studies in Immunology. Garland Science, USA.

[8] Marks, F., Klingmüller, U. and Müller-Decker, K. (2009) Cellular Signal Processing, Garland Science, USA.

[9] Molina-Paris, C. and Lythe, G. (2011) Mathematical Models and Immune Cell Biology. Springer, New York. https://doi.org/10.1007/978-1-4419-7725-0

[10] Murphy, K. (2012) Immuno Biology. 8th Edition, Garland Science, USA.

[11] Rees, R.C. (2014) Tumor Immunology and Immunotherapy. Oxford University Press, Oxford.

[12] Cheng, F.H.C. and Agudas, B.D. (2014) A Mathematical Model of Bimodal Epigenetics Control of miR-193a in Ovarian Cancer Stem Cells. PLoS ONE, 9, e116050.

[13] Lukianova-Hleb, E.Y. and Lapotko, D.O. (2015) Rapid Detection and Destruction of Squamous Cell Carcinoma of the Head and Neck by Nano-Quadrapeutics. Head Neck, 37, 1547-1555. https://doi.org/10.1002/hed.24018

[14] Lukianova-Hleb, E.Y., Ren, X., Sawant, R.R., Wu, X., Torchilin, V.P. and Lapotko, D.O. (2014) On-Demand Intracellular Amplification of Chemoradiation with Cancer-Specific Plasmonic Nanobubbles. Nature Medicine, 20, 778-784. https://doi.org/10.1038/nm.3484

[15] Lukianova-Hleb, E., Hu, Y., Latterini, L., Tarpani, L., Lee, S., Drezek, R.A., Hafner, J.H. and Lapotko, D.O. (2010) Plasmonic Nanobubbles as Transient Vapor Nanobubbles Generated around Plasmonic Nanoparticles. ACS Nano, 4, 2109-2123. https://doi.org/10.1021/nn1000222

[16] Larsen, J.C. (2016) The Bistability Theorem in a Model of Metastatic Cancer. Applied Mathematics, 7, 1183-1206.

[17] Larsen, J.C. (2016) The Bistability Theorem in a Cancer Model. Preprint.

[18] Horn, F. and Jackson, R. (1972) General Mass Action Kinetics. Archive for Rational Mechanics and Analysis, 47, 81-116. https://doi.org/10.1007/BF00251225

[19] Uherka, D.J. and Sergott, A.M. (1977) On the Continuous Dependence of the Roots of a Polynomial on Its Coefficients. The American Mathematical Monthly, 84, 368370. https://doi.org/10.2307/2319971

[20] Nylandssted, J., et al. (2014) S100A11 Is Required for Efficient Plasma Membrane Repair and Survival of Invasive Cancer Cells. Nature Communications, 5, Article No. 3795. 
Submit or recommend next manuscript to SCIRP and we will provide best service for you:

Accepting pre-submission inquiries through Email, Facebook, LinkedIn, Twitter, etc. A wide selection of journals (inclusive of 9 subjects, more than 200 journals)

Providing 24-hour high-quality service

User-friendly online submission system

Fair and swift peer-review system

Efficient typesetting and proofreading procedure

Display of the result of downloads and visits, as well as the number of cited articles Maximum dissemination of your research work

Submit your manuscript at: http://papersubmission.scirp.org/

Or contact am@scirp.org 